\title{
The fine-structure contamination of vertical velocity spectra in the deep ocean*
}

\author{
G. SIEDLER $\dagger$
}

(Received 13 April 1973; in revised form 9 August 1973; accepted 23 August 1973)

\begin{abstract}
When determining vertical velocity spectra from temperature time series and the mean vertical temperature gradient, restrictions may arise from the existence of fine-structure. PHILLIPs (1971) and GARRETT and MUNK (1971) have shown that the fine-structure contamination of internal gravity wave spectra can be written as a function of some statistical properties of the internal wave field and the vertical wave number spectrum of the fine-structure. A consistent set of current and temperature data was obtained during an experiment at Site $D$ to study this problem. The wave number spectrum of the vertical temperature fine-structure and the apparent frequency spectrum of internal waves are determined from these data. In contrast to the assumptions in the above models, our fine-structure data imply a wave number spectrum proportional to (wave number) ${ }^{-3}$ in the range which is important here. Using the above set of data, a model is suggested to describe the effect of fine-structure on vertical velocity spectra computed with the mean vertical temperature gradient. It indicates a maximum finestructure contamination of the true frequency spectrum of internal gravity waves in the middle of the internal wave band, with less contamination at low and high frequencies.
\end{abstract}

\section{INTRODUCTION}

TIME SERIES of vertical velocity in the deep ocean can be obtained either by direct measurements with floats or indirectly from moored temperature meters.

The most successful attempt to measure vertical velocities directly was based on Webb's instrument (VoORHIS, 1968; WeBB and WortHINGTON, 1968; VoORHIS and WEBB, 1970). There, a float with an array of tilted vanes is used which is balanced to stay at a predetermined depth. A vertical current turns the float around its vertical axis, and the rate of rotation indicates the magnitude of vertical velocity. It can be determined with the aid of a magnetic compass reference. The data are recorded inside the instrument or transmitted to a ship acoustically.

Unfortunately, it is yet impossible to obtain time series over more than a few days by this technique. Furthermore, it is often desirable to obtain horizontal and vertical velocities simultaneously at the same position. Although the horizontal motion of the above float can be determined by using acoustic transponders, the resolution in time and space is very restricted. When there is a need for long simultaneous time series of horizontal and vertical velocity with a high resolution in time, temperature measurements by moored instruments are the only method available to determine vertical velocities in the deep ocean.

\section{VERTICAL VELOCITIES DEDUCED FROM TEMPERATURE VARIATIONS}

The time variation of the temperature of a water particle can be described by

$$
\mathrm{d} T / \mathrm{d} t=\partial T / \partial t+u \partial T / \partial x+v \partial T / \partial y+w \partial T / \partial z,
$$

*Contribution No. 3067 from the Woods Hole Oceanographic Institution.

†Institut für Meereskunde, Kiel Universität, Düsternbrooker Weg 20, 23 Kiel, Germany. 


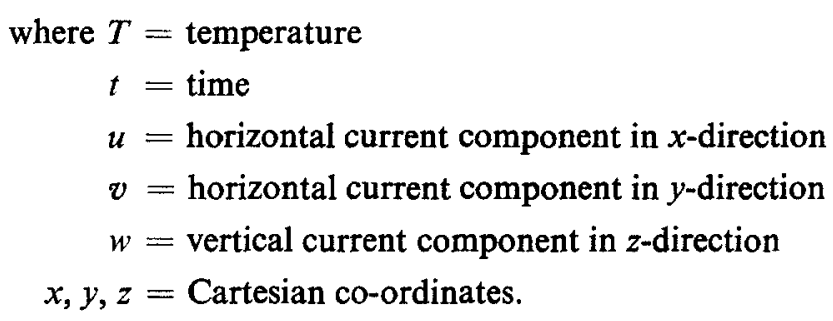

If $T$ were a conservative property, the expression in (1) would equal zero. For long-term changes this is not the case; diffusion will change the heat content of a water particle. Close to the surface, additional heat sinks and sources due to air-sea interaction will cause temperature variations even on short time scales. Thus the assumption of temperature being a conservative property will only be a good approximation for short-term changes in the deep ocean. The following discussion will therefore be restricted to the short-term variability in the internal gravity wave band in the deep ocean, and the magnitude of the vertical displacement will be assumed small enough to neglect adiabatic temperature changes.

Furthermore, the two terms in (1) connected to horizontal advection will be neglected against the term containing vertical motion. This is possibly a poor approximation in areas with large horizontal temperature gradients.

With the above assumptions one obtains

$$
w=-\left(\frac{\partial T}{\partial z}\right)^{-1} \frac{\partial T}{\partial t} .
$$

Unfortunately, vertical variations in $\partial T / \partial z$ usually cover scales down to values smaller than the scale of vertical displacements due to internal waves (e.g. SIEDLER, 1969; OSBORN and CoX, 1972). Therefore, the vertical temperature gradient is more adequately described by a mean gradient $\overline{\partial T} / \partial z$ and a fine-structure gradient $\partial \theta / \partial z$ :

$$
\partial T / \partial z=\overline{\partial T} / \partial z+\partial \theta / \partial z \text {. }
$$

The averaging interval is chosen in a way which leads to $\overline{\partial T} / \partial z=$ const. and $\overline{\partial \theta} / \partial z=0$ for scales somewhat larger than internal wave displacements. The available data indicate that the fine-structure varies sufficiently slowly in time and horizontal space to use the frozen fine-structure assumption for the following discussion (GARRETT and MUNK, 1971 ; OsBORN and Cox, 1972).

It follows from (2) and (3):

$$
w=-\frac{\partial T / \partial t}{\partial T / \partial z+\partial \theta / \partial z}
$$

The usual moored current/temperature meters do not measure the local temperature gradient. The mean gradient $\overline{\partial T} / \partial z=T^{\prime}$ can, however, be determined from hydrographic profiling, and $w$ is usually calculated by neglecting $\partial \theta / \partial z$ in (4):

$$
w \sim-\left(T^{\prime}\right)^{-1} \partial T / \partial t
$$

The following discussion is an attempt to estimate the fine-structure contamination of the vertical velocity frequency spectrum when $w$ is calculated from (5). 


\section{EARLIER FINE-STRUCTURE MODELS}

In his paper on spectra measured in an undulating layered medium, PHILLIPS (1971) showed that a step-like structure of layers and sheets introduces a contribution proportional to $\omega^{-2}$ ( $\omega=$ angular frequency) to an internal wave spectrum, and REID (1971) gave the corresponding solution for the special case of one step dividing two homogeneous layers.

GARRETT and MUNK (1971) treated the problem for the more general case where the statistics of the layering is prescribed. They obtain the result that for a vertically homogeneous fine-structure field and a Gaussian wave field the frequency spectrum can be obtained if the fine-structure scale $K^{-1}$ is small compared to the r.m.s. wave displacement $Z$, or:

$$
K Z \gg 1 \text {. }
$$

Here $K$ is determined from the fine-structure spectrum $F_{\theta}(k)$ as a function of wave number $k$ by:

$$
K^{2}=\frac{\int_{k_{L}}^{k_{s}} k^{2} F_{\theta}(k) \mathrm{d} k}{\int_{k_{L}}^{k_{s}} F_{\theta}(k) \mathrm{d} k} .
$$

$k_{L}$ and $k_{S}$ are some lower and upper wave number limits which are identified with layers and sheets in the case of a step-like fine-structure. As will be seen later, this 'fine-structure approximation' may well be a poor approximation because it is difficult to find a lower wave number limit for the fine-structure.

According to equation (16) in GARRETT and MUNK's paper (1971), the part of the auto-spectrum $F_{T}{ }^{f s}(\omega)$ of temperature which is due to fine-structure superimposed on a mean gradient in the presence of an internal wave field can be written as:

$$
F_{T^{f}}{ }^{f}(\omega)=\int_{0}^{\infty}\left(\frac{2}{\pi}\right)^{\frac{1}{2}} S Z F_{\theta^{\prime}}[k(p)] \exp \left(-\omega^{2} p\right) \mathrm{d} p .
$$

Here, $F_{\theta}^{\prime}(k)=k^{2} F_{\theta}(k)$ is the fine-structure gradient spectrum and $F_{\theta}(k)$ is the finestructure spectrum. The wave number $k$ and the variable $p$ are related by:

$$
k=[S Z \sqrt{ }(2 p)]^{-1} \text {. }
$$

The internal wave frequency scale $S$ is given by:

$$
S^{2}=\frac{\int_{f}^{N} \omega^{2} F_{\zeta}(\omega) \mathrm{d} \omega}{\int_{f}^{N} F_{\zeta}(\omega) \mathrm{d} \omega} .
$$

Here $f$ and $N$ are the inertial and the Brunt-Väisälä frequency, $F_{\zeta}(\omega)$ is the spectrum of the displacement $\zeta$. Garrett and Munk discuss the results of their theory for two fine-structure models, one with a $k^{-2}$ slope over the range $k_{L} \leq k \leq k_{S}$ and zero otherwise, and another one with a steady decrease down to zero below $k_{L}$. Both models yield similar results. In the later discussion, the problem will be treated along their line of 
arguments, but the fine-structure model and the internal wave model will be based on wave number and frequency spectra obtained during a specific experiment.

\section{THE DATA}

Two moorings were placed at Site $\mathrm{D}\left(\psi=39^{\circ} 08^{\prime} \mathrm{N}, \lambda=69^{\circ} 59^{\prime} \mathrm{W}\right)$ for nine days. The horizontal separation was $920 \mathrm{~m}$, and the water depth was approximately $2660 \mathrm{~m}$. Current/temperature meters were placed at three levels $(533,583,633 \mathrm{~m})$. During oneday periods immediately after launching the second mooring and three days before retrieving both moorings, additional data were obtained on hydrographic stations and STD-stations with repeated lowerings covering the depth range of the moored instruments. The lowering speed of the STD was adjusted with respect to the response time of the temperature sensor to obtain a maximum vertical resolution of about $1 \mathrm{~m}$. The primary purpose of this experiment was a study of spatial scales of internal waves whose results will be published elsewhere.

The experiment supplied a set of simultaneous data for a certain time period and a selected area in the deep ocean which can now be used to study the fine-structure contamination of internal wave spectra. The data from six STD-stations with 4, 6 or 8 repeated profiles each were used to compute wave number spectra $F_{\theta}(k)$ for the depth range $480-680 \mathrm{~m}$ or 440-600 m. Pre-whitening methods were used in the analysis. The spectra are shown in Fig. 1. A visual inspection indicates no significant changes from one set of profiles to the next one. All estimates of a total of 36 profiles for a depth range $440-600 \mathrm{~m}$ were then averaged, and the resulting spectrum is plotted in Fig. 2. The

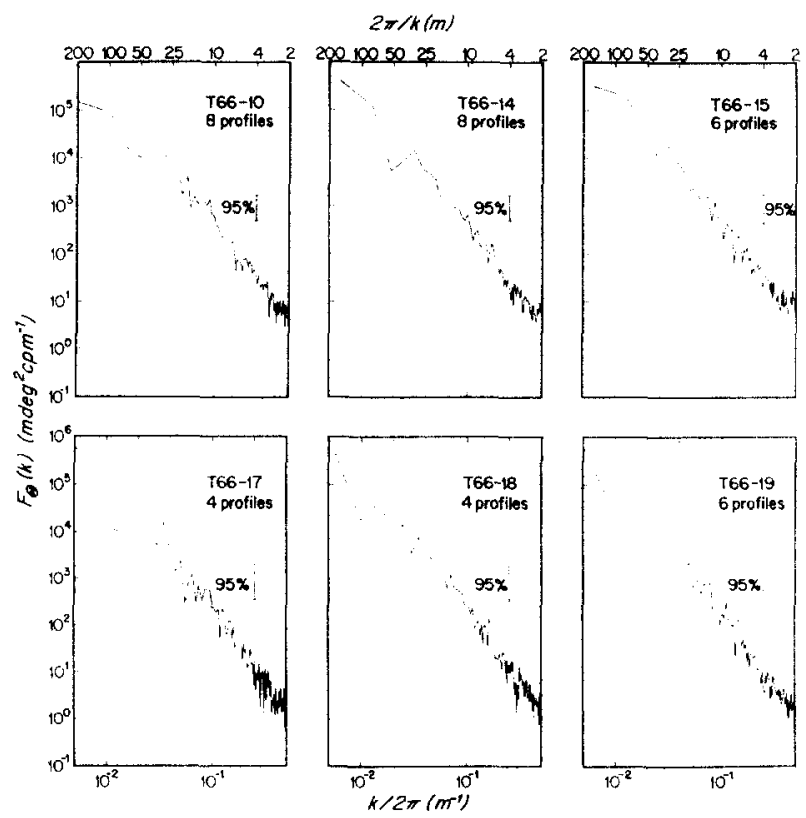

Fig. 1. Vertical wave number spectra of temperature fine-structure obtained from repeated STD lowerings at 6 stations, estimates averaged from spectra for 8,6 or 4 profiles each. 


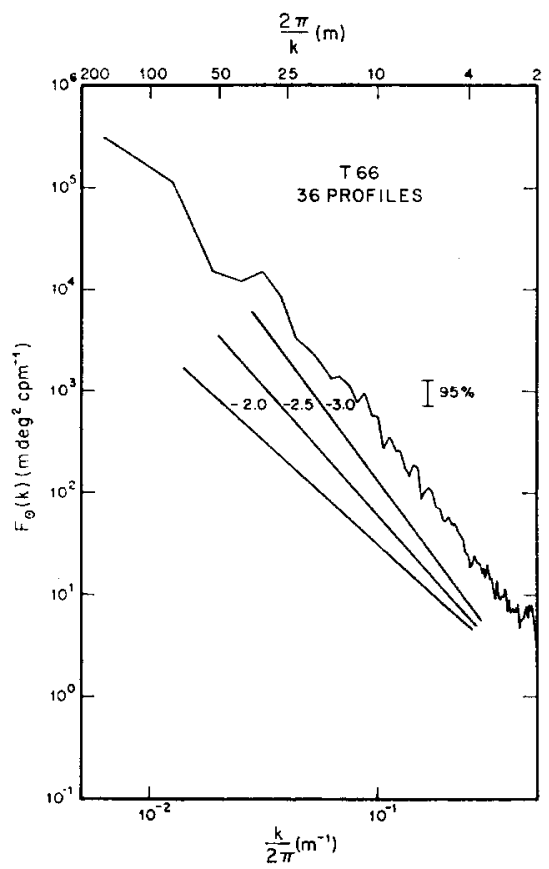

Fig. 2. Vertical wave number spectrum of temperature fine-structure obtained by averaging estimates from 36 spectra of individual profiles.

spectrum can be described by a wave number dependence proportional to $k^{-q}$ where $q$ is between $2 \cdot 5$ and 3 and can be reasonably well approximated by 3 over a wide range. Somewhat similar results were obtained by R. Millard (personal communication) who observed a slope between -2.5 and -3 in the spectrum $F_{\theta}(k)$ at corresponding wave numbers, with the energy level decreasing for decreasing mean gradient. The temperature gradient spectrum $F_{\theta^{\prime}}(k)$ given by OsBoRN and Cox (1972) leads to $F_{\theta}(k)$ proportional to $k^{-2 \cdot 3}$ up to $k^{-4}$ for wave numbers between $10^{-3}$ and $10^{-2}$ cycles $\mathrm{cm}^{-1}$. RoDEN's (1971) spectra probably do not display such steep slopes because data from depth ranges with very different mean gradients are combined there.

\section{THE FINE-STRUCTURE CONTAMINATION}

The data from the above experiment suggest a fine-structure spectrum $F_{\theta}(k)$ proportional to $k^{-3}$ or a gradient spectrum $F_{\theta}(k)$ proportional to $k^{-1}$ over a range of wave numbers from approximately $2 \pi / 50 \mathrm{~m}^{-1}$ to at least $2 \pi / 2 \mathrm{~m}^{-1}$. The limits are not well defined, and the effect of extending the range with the steep slope to lower wave numbers will be discussed later. A fine-structure model similar to GARRETT and MUNK's (1971) model is used, but with the important difference of a steeper slope in the wave number spectrum:

$$
\begin{array}{ll}
\text { Range 1: } F_{\theta^{\prime}}(k)=0 & \text { for } k<k_{L} \\
\text { Range 2: } F_{\theta^{\prime}}(k)=\frac{\overline{\theta^{\prime 2}}}{\ln k_{S}-\ln k_{L}} k^{-1} & \text { for } k_{L} \leq k \leq k_{S} \\
\text { Range 3: } F_{\theta}^{\prime}(k)=0 & \text { for } k_{S}<k .
\end{array}
$$


Here $\overline{\theta^{\prime 2}}$ is the variance of the fine-structure gradient.

Inserting (11) into (8) leads to the following expression where the slashes in the brackets indicate the limits corresponding to $k_{L}$ and $k_{S}$ :

$$
F_{T} f^{f}(\omega)=2 \pi^{-\frac{1}{1}} S^{2} Z^{2} \frac{\overline{\theta^{\prime 2}}}{\ln k_{S}-\ln k_{L}} \int_{0}^{\infty}\left[0 / p^{\ddagger} / 0\right] \exp \left(-\omega^{2} p\right) \mathrm{d} p .
$$

By carrying out the Laplace transformation, we obtain:

$$
F_{T}^{f s}(\omega)=S^{2} Z^{2} \frac{\overline{\theta^{\prime 2}}}{\ln k_{S}-\ln k_{L}} \omega^{-3}\left[\exp \left(-\omega^{2} \omega S^{-2}\right)-\exp \left(-\omega^{2} \omega_{L}{ }^{-2}\right)\right]
$$

with

$$
\omega_{S, L}=S Z k S, L / \sqrt{ } 2 .
$$

The essential difference to GARRETT and MUNK (1971) is the $\omega^{-3}$ instead of $\omega^{-2}$ term in the above equation. The function in brackets in (13) behaves like

$$
\begin{array}{ll}
\omega^{2} \omega_{L}-2 & \text { for } \omega \ll \omega_{L} \\
1 & \text { for } \omega_{L} \ll \omega \ll \omega_{S} \\
\exp \left(-\omega^{2} \omega_{S^{-2}}\right) & \text { for } \omega_{S} \ll \omega .
\end{array}
$$

With a temperature spectrum $F_{T}^{g d}(\omega)=T^{\prime 2} F_{5}(\omega)$ due to the mean gradient $T^{\prime}$ and an internal wave field with a vertical displacement spectrum $F_{\zeta}(\omega)$ we are now able to calculate the ratio $\gamma$ :

$$
\gamma=\frac{F_{T^{f s}}(\omega)}{F_{T^{g d}}(\omega)}
$$

A ratio $\gamma$ close to 1 or larger would indicate fine-structure contamination. The ratios $\gamma_{i}$ for the above three ranges $i=1,2,3$ are given by:

$$
\begin{aligned}
& \gamma_{1}=\gamma_{2} \frac{\omega^{2}}{2 S^{2} Z^{2} k_{L}^{2}} \\
& \gamma_{2}=\frac{S^{2} Z^{2} \overline{\theta^{\prime 2}} \omega^{-3}}{T^{\prime 2} F_{6}(\omega)\left(\ln k_{S}-\ln k_{L}\right)} \\
& \gamma_{3}=\gamma_{2} \exp \left(-\frac{\omega^{2}}{2 S^{2} Z^{2} k_{S^{2}}}\right) .
\end{aligned}
$$

To compute the $\gamma_{i}$, we need some information about the internal wave field. The autospectra $F_{u, v}(\omega)$ of horizontal velocity between $f$ and $N$ are nearly proportional to $\omega^{-2}$ (Figs. 3a-d). If they are dominated by internal waves, linear internal wave theory leads to a spectrum $F_{\zeta}(\omega)$ proportional to $\omega^{-2}$ and a vertical velocity spectrum $F_{w}(\omega)$ which is flat in the internal wave range. Furthermore, the estimates of horizontal and vertical velocity should be equal on the average at a frequency $N / \sqrt{ } 2$ (PochapsKy, 1972).

The vertical velocity spectrum $F_{w v^{j d}}(\omega)=T^{\prime-2} F_{\partial T / \partial t}(\omega)$ was computed from the observed temperature difference spectrum $F_{\partial T / \partial t}(\omega)$ and the observed mean gradient $T^{\prime}$, and the resulting spectra for instruments $4182,4184,4185$ and 4193 are plotted on top of the spectra $F_{u, v}(\omega)$ of horizontal velocity in Figs. 3a-d. The inertial frequency at Site $D$ is $0.053 \mathrm{cph}$, the local Brunt-Väisälä frequency at the selected depth is approximately $1 \mathrm{cph}$. It turns out that $F_{w}{ }^{g d}(\omega)$ has a mean slope much smaller than 1 between 


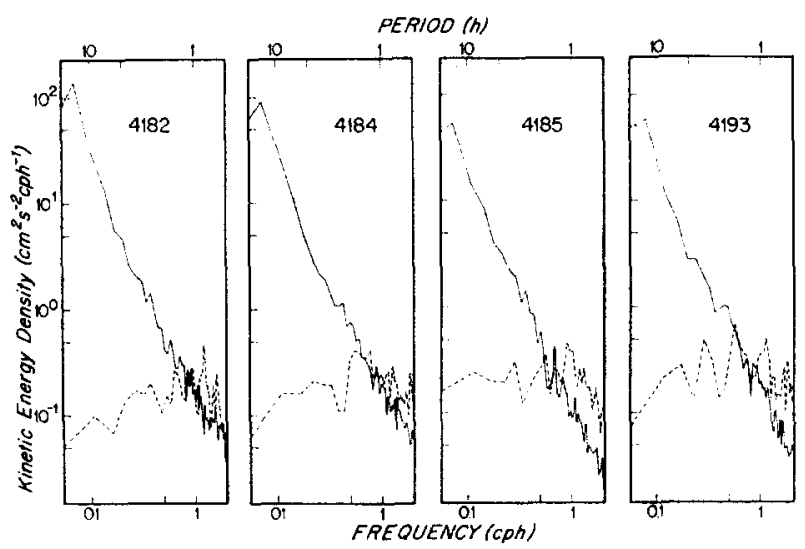

Fig. 3. Frequency spectra of horizontal velocity obtained from current measurements (solid lines) and of vertical velocity from temperature measurements with the same instruments when applying mean temperature gradients from STD-profiling (dotted lines). Depth levels are $533 \mathrm{~m}$ (No. 4182), $583 \mathrm{~m}$ (Nos. 4184 and 4193$)$ and $633 \mathrm{~m}$ (No. 4185).

$f$ and $N$ and drops off to low values below and above these limiting frequencies. Straight horizontal lines through the flat portions of $F_{w}{ }^{g d}(\omega)$ cross the smoothed spectra $F_{u, v}(\omega)$ near $N / \sqrt{ } 2=0.7 \mathrm{cph}$.

It therefore seems reasonable to assume that these spectra resemble mostly an internal wave field, and that fine-structure contamination does not increase the mean energy density level of $F_{w}{ }^{\sigma d}(\omega)$ by one order of magnitude or more.

We then write the true displacement spectrum with the constant $A$ as $F_{5}(\omega) \sim A \omega^{-2}$ for $f \leq \omega \leq N$ and zero otherwise. The fine-structure spectrum is described by $F_{\theta^{\prime}}(k) \sim E k^{-1}$ for $k_{L} \leq k \leq k_{S}$ and zero otherwise.

We obtain within the above limits for $\omega$ and $k$ :

$$
\begin{aligned}
& S^{2}=\frac{\int_{f}^{N} \omega^{2} F_{\zeta}(\omega) \mathrm{d} \omega}{\int_{f}^{N} F_{\zeta}(\omega) \mathrm{d} \omega} \sim \frac{\int_{f}^{N} A \mathrm{~d} \omega}{\int_{f}^{N} A \omega^{-2} \mathrm{~d} \omega} \sim N f \\
& Z^{2}=\int_{f}^{N} F_{\zeta}(\omega) \mathrm{d} \omega \sim \int_{f}^{N} A \omega^{-2} \mathrm{~d} \omega \sim \frac{A}{f} \\
& \overline{\theta^{\prime 2}}=\int_{k_{L}}^{E_{g}} k^{-1} \mathrm{~d} k=E\left(\ln k_{S}-\ln k_{L}\right) .
\end{aligned}
$$

Inserting (15) in (14) results in

$$
\left.\begin{array}{l}
\gamma_{1}=\frac{\overline{\theta^{\prime 2}}}{T^{\prime 2}\left(\ln k_{S}-\ln k_{L}\right) 2 k_{L}{ }^{2} A} \omega \\
\gamma_{2}=\frac{N \overline{\theta^{\prime 2}}}{T^{\prime 2}\left(\ln k_{S}-\ln k_{L}\right)} \omega^{-1} \\
\gamma_{3}=\frac{N \overline{\theta^{\prime 2}}}{T^{\prime 2}\left(\ln k_{S}-\ln k_{L}\right)} \omega^{-1} \exp \left(-\frac{\omega^{2}}{2 k_{S^{2} A N}}\right) .
\end{array}\right\}
$$


From our data, we find with $k_{L}=2 \pi / 50 \mathrm{~m}^{-1}$ and $k_{S}=2 \pi / 2 \mathrm{~m}^{-1}$

$$
\begin{aligned}
& S^{2}=0.053 \times 4 \pi^{2} \mathrm{~h}^{-2} \\
& Z^{2}=119 \mathrm{~m}^{2} \\
& A=6.25 \times 2 \pi \mathrm{m}^{2} \mathrm{~h}-1 \\
& E=0.5 \times 4 \pi^{2}\left(10^{-3} \mathrm{C}^{2} \mathrm{~m}^{-2}\right. \\
& \overline{\theta^{\prime 2}}=64\left(10^{-3}{ }^{\circ} \mathrm{C}\right)^{2} \mathrm{~m}^{-2} \\
& \left(T^{1}\right)^{-2}=0.0625\left(10^{-2}{ }^{\circ} \mathrm{C}\right)^{-2} \mathrm{~m}^{2} \text { at } z=583 \mathrm{~m} .
\end{aligned}
$$

The ratio of the mean square fine-structure variation to the square of the mean gradient is 4 . If very high wave numbers were to be considered, this ratio would be higher (GREGG and CoX, 1972). Because of a very large $\omega_{S}$, this is not necessary in this context.

Using the above numbers, we find (dimensions of $\omega / 2 \pi=h^{-1}$ ):

$$
\left.\begin{array}{l}
\gamma_{1}(\omega)=6 \cdot 3 \omega / 2 \pi \\
\gamma_{2}(\omega)=1 \cdot 2 \omega^{-1} / 2 \pi,
\end{array}\right\}
$$

and

$$
\begin{aligned}
& \omega_{L} / 2 \pi=0.45 \mathrm{cph} \\
& \omega_{S} / 2 \pi=11 \mathrm{cph} .
\end{aligned}
$$

\section{CONCLUSIONS}

The result is sketched in Fig. 4. Here $\hat{F}_{w^{g d}}(\omega)$ is the true vertical velocity spectrum, normalized with respect to energy density to equal 1 in the internal wave band. The normalized fine-structure spectrum $\hat{F}_{w}{ }^{f s}(\omega)$ equals $\gamma(\omega)$ between $f$ and $N$.

The spectrum obtained from temperature time series in the presence of fine-structure is given by $\hat{F}_{w^{g d+f s}}(\omega)$. For a comparison $\hat{F}_{w G M}{ }^{f s}(\omega)$ and $\hat{F}_{w G M}{ }^{g d+f s}(\omega)$ indicate the respective spectra as obtained from GARRETT and MUNK's model for the same Coxnumber $C$ :

$$
C^{2}=\overline{\theta^{\prime}} / T^{\prime 2} \sim 4
$$

It can be concluded from our data that the fine-structure contamination has a maximum at $\omega_{L}$ and is smaller at lower and higher frequencies. A smaller $K_{L}$ shifts the maximum of the fine-structure contamination towards lower frequencies and increases its magnitude. Because $\omega_{S}$ turns out to be much larger than $N$ in our case, the fine-structure at wave numbers close to the lower limit $k_{L}$ is most important.

With a flat spectrum for $\theta^{\prime}$ as assumed by GARRETT and MUNK (1971) and the same Cox-number and r.m.s. wave displacement, we find a maximum of contamination at the highest internal wave frequencies. The considerably lower level of $\hat{F}_{w G M^{f 8}}$ relative to $\hat{F}_{w} f_{8}$ is due to the smaller fine-structure contribution to temperature variance in this case. The relative contribution by the fine-structure to the temperature variance produced by internal waves is given by $C^{2} D^{2}$ [see GARRETT and MUNK (1971), equation (26)] where the Fine-Structure Number $D$ is defined as

$$
D^{2}=\left(K^{2} Z^{2}\right)^{-1} \text {. }
$$




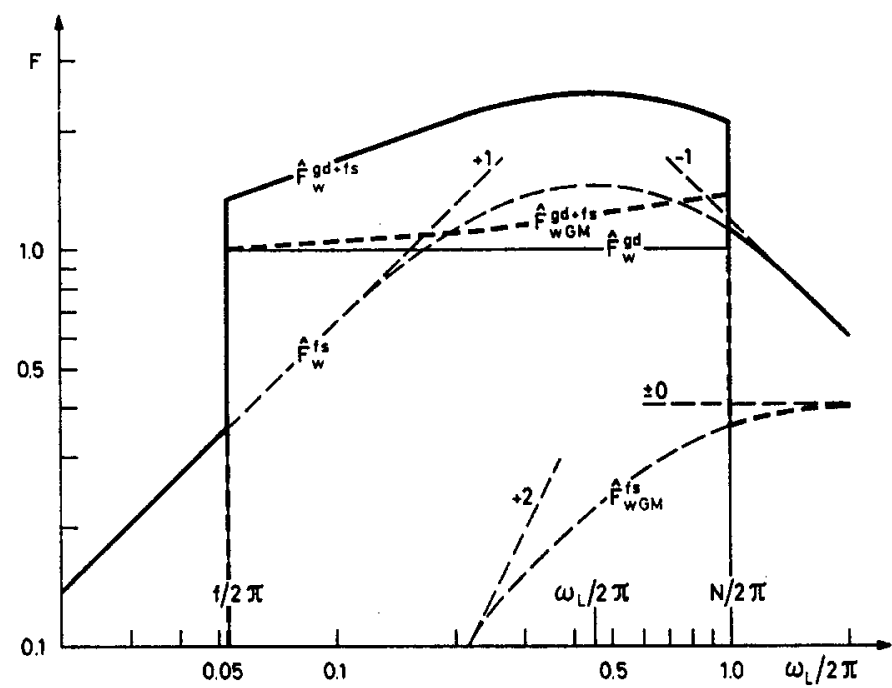

Fig. 4. Schematic presentation of fine-structure contamination in normalized vertical velocity spectra.

$\hat{F}_{w}$ od: spectrum obtained from temperature series in the presence of a mean temperature

gradient only.

$\hat{F}_{\text {wot }}$ : spectral contribution due to fine-structure.

$f_{w^{o d+f s}}$ : contaminated spectrum.

For a comparison, the respective spectra from GARRETT and MUNK's (1971) model for the same

Cox-number are indicated by $\hat{F}_{w \mathrm{GM}^{f s}}$ and $\hat{F}_{w \mathrm{GM}}{ }^{p d+f_{s}}$. The numbers indicate the slopes of the approximating functions $\gamma_{1}$ and $\gamma_{2}$.

In our case, $K^{2} Z^{2} \sim 4$, and therefore $C^{2} D^{2} \sim 1$ while Garrett and Munk's model with the same $C$ and $Z$ leads to $K^{2} Z^{2} \sim 47$ and $C^{2} D^{2} \sim 0.09$.

Assuming that the basic model provides a reasonable approximation, although $K Z$ is not much larger than 1 in our case, and considering that the wave number spectra $F_{\theta^{\prime}}(k)$ are proportional to $k^{-n}$ with $n$ close to, but somewhat below 1 , the actual contamination and the resulting spectrum $\hat{F}_{w}{ }^{g d+f s}(\omega)$ will be somewhat below the values indicated in Fig. 4. The observed spectra in Fig. 3 seem to indicate a trend similar to

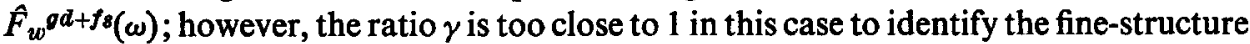
contributions in these spectra directly.

Acknowledgements-This work was supported by the Office of Naval Research, Contract No. N0001466-C0241. The experiment at sea and the data evaluation was carried out with the aid of the Buoy Group and of P. M. SAUNDERS at the Woods Hole Oceanographic Institution. I particularly benefited from discussions with N. P. Fofonoff, K. Hasselmann, T. Joyce, R. Millard, JR. and F. Webster.

\section{REFERENCES}

GARRETT C. and W. MUNK (1971) Internal wave spectra in the presence of fine-structure. Journal of Physical Oceanography, 1(3), 196-202.

GREGG M. C. and C. S. Cox (1972) The vertical microstructure of temperature and salinity. Deep-Sea Research, 19(5), 355-376.

OsBorn T. R. and C. S. Cox (1972) Oceanic fine structure. Geophysical Fluid Dynamics, 3, 321-345.

PhillIPs O. M. (1971) On spectra measured in an undulating layered medium. Journal of Physical Oceanography, 1(1), 1-6. 
POCHAPSKY T. E. (1972) Internal waves and turbulence in the deep ocean. Journal of Physical Oceanography, 2(1), 96-103.

REID R. O. (1971) A special case of Phillips' general theory of sampling statistics for a layered medium. Journal of Physical Oceanography, 1(1), 61-62.

RODEN G. I. (1971) Spectra of North Pacific temperature and salinity perturbations in the depth domain. Journal of Physical Oceanography, 1(1), 25-33.

SIEDLER G. (1969) On the fine structure of density and current distribution and its short-time variations in different areas. Progress in Oceanography, Mary SEars, editor, Pergamon Press, 5, 81-94.

Voorirs A. D. (1968) Measurements of vertical motion and the partition of energy in the New England Slope Water. Deep-Sea Research, 15, 599-608.

VOORHIS A. D. and D. C. WEBB (1970) Large vertical currents observed in a winter sinking region of the Northwestern Mediterranean. Cahiers Océanographiques, 22, 571-580.

WEBB D. C. and L. V. WORTHINGTON (1968) Measurements of vertical water movement in the Cayman Basin. Deep-Sea Research, 15, 609-612. 